\title{
MITARBEITERGESPRÄCHE
}

\section{Wer fragt, der führt}

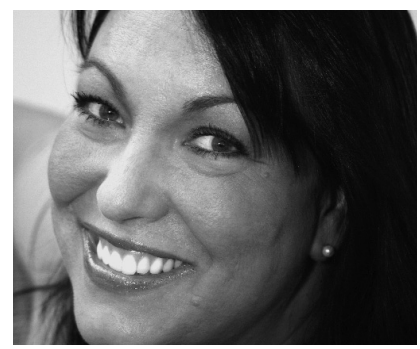

VON ANKE BALLMANN

Anke Ballmann ist Pädagogin mit einer eigenen Praxis für Pädagogische Psychologie mit Spezialisierung auf Diagnostik, Beratung und Förderung. Als Referentin ist sie in der Weiterbildung für Organisationen und Verbände tätig. Internet http://www.lernmeer.de

\section{Konfliktlösungen erfordern Übung und das gilt beson- ders für schwierige Gespräche. Sie benötigen eine ande- re Art der Gesprächsführung als das tägliche Sprechen miteinander. Sollen Konfliktgespräche erfolgreich ver- laufen, müssen sie gut geplant und vorbereitet werden.}

Im Führungsalltag werden Leitungskräfte immer wieder mit Gesprächssituationen konfrontiert, die herausfordern. Sei es in den jährlichen Beurteilungs- und Zielvereinbarungsgesprächen oder wenn Mitarbeitende ein zu positives Selbstbild haben. Geraten schwierige Gespräche aus dem Ruder, kann es viel Zeit und Energie kosten, um eine effektive Zusammenarbeit wiederherzustellen. Deshalb ist es klüger, Gespräche geschickt zu gestalten, denn dann steigt die Mitarbeiterzufriedenheit und Unternehmensziele werden leichter erreicht. Zwei Aspekte sind dabei besonders bedeutsam:

- Offene Fragen stellen: Von dem griechischen Philosophen Sokrates ist überliefert: "Wer fragt, der führt das Gespräch. «In der Tat sind gut gestellte Fragen die wichtigste und effektivste Technik der Gesprächsführung. Für schwierige Gespräche eignen sich besonders offene W-Fragen: wann, warum, wieso, weshalb, wo, wovon, wovor, woran, wer, wie, wie genau, in welcher Weise, woran, inwieweit, was etc. Antworten auf solche Fragen enthalten in der Regel viele Informationen, da die Frage nicht mit einem knappen Ja oder Nein beantwortet werden kann.

- Aktiv Zuhören: Als »aktives Zuhören « bezeichnet man die Gesprächstechnik, die Aussagen der Gesprächspartner sinngemäß zu wiederholen. Durch aktives $\mathrm{Zu}$ - hören soll erreicht werden, dass der Sprecher die Gewissheit hat, dass der Zuhörer auf ihn eingeht: Er soll sich ernst genommen fühlen! Weiterhin soll der Zuhörer die Anliegen und Bedürfnisse des Gesprächspartners besser verstehen. Missverständnisse in der Kommunikation werden so schneller sichtbar und können ausgeräumt werden.

\section{Kooperation als Königsweg zur Konfliktlösung}

Bei der kooperativen Konfliktbewältigung geht es nicht um »Schuld «, »Recht haben " oder "gewinnen ", sondern um das gemeinsame Bearbeiten und Lösen eines Problems. Kooperation ist lösungsorientiert, das heißt: Die Konfliktpartner treten sich nicht wie zwei Gegner gegenüber, sondern erarbeiten zusammen Lösungsmöglichkeiten.

Kommunikationsregeln dabei sind:

- Gesprächspartners und seine Persönlichkeit achten: Nur sachliche Kritik vortragen.

- Keine sachliche Vorwürfe, nicht verletzen.

- Kein Gesprächspartner soll dem anderen ins Wort fallen, ausreden lassen.

- Probleme des Gesprächspartners ernst nehmen: Nichts herunterspielen.

- Gemeinsam nach Lösungen suchen: Keine Lösungen aufdrängen. 
Die Phasen eines Kooperationsgesprächs sind oft:

- Eröffnungsphase: angenehme Atmosphäre schaffen, Ziel des Gesprächs klären, Zeitrahmen abstecken, Kommunikationsregeln vereinbaren

- Themenphase: Sammlung der Themen, jeder Partner schildert den Konflikt aus seiner Sicht. Es gibt keine Anschuldigungen, kein Unterbrechen, keine sofortige Suche nach Lösungen!

- Herausarbeiten der Interessen und Bedürfnisse: Jeder schildert, was ihm an dem Thema wichtig ist.

- Lösungsphase: Brainstorming, sammeln und gemeinsame Auswahl von Lösungen.

- Vereinbarungen treffen und festlegen, wie ihre Einhaltung zu überprüfen ist.

Beispiele für Fragen, die Kooperation erleichtern:

- Wie sehen sie die Situation?

- Was sind ihrer Ansicht nach die Ursachen des Konflikts?

- Was können wir tun, um wieder besser zusammenzuarbeiten?

- Können wir die getroffenen Vereinbarungen akzeptieren?

\section{Was Sie tun können, wenn der Kooperation Gefahr droht ...}

Bei Unverschämtheiten: Im Eifer eines Gesprächs können unangemessene Formulierung herausrutschen. Wenn Sie Ihren Mitarbeiter oder Ihre Mitarbeiterin dann auffordern das Gesagte zu wiederholen, nehmen Sie das Spontane aus der Situation heraus.

Bei Aggressionen: Schweigen ist eine harte, jedoch wirksame Form der Deeskalation. Ihr Mitarbeiter rechnet mit einer Reaktion von Ihnen. Wenn Sie sich nicht erwartungsgemäß verhalten, verunsichern Sie Ihr Gegenüber taktvoll. Blicken Sie Ihrem Gegenüber fest in die Augen, und schweigen Sie bedeutsam.

Bei Volltreffern: Wenn Sie sich durch die Äußerung eines Mitarbeiters getroffen fühlen, sollten Sie das ansprechen. Ein gewolltes Understatement der eigenen Persönlichkeit ist ein wunderbarer rhetorischer Schachzug. Der Trick dabei ist, die Verletztheit ironisch, lächelnd und freundlich vorzu- bringen. Durch Ihre unerwartete Botschaft verunsichern Sie Ihren Gesprächspartner und Verunsicherungen sind gut um neue Ideen hervorzubringen.

\section{Im Notfall}

Geben Sie großer Wut auf gar keinen Fall nach! Auf Angriffe reagieren die meisten Menschen intuitiv mit einem Gegenangriff. Verständlich, aber nicht kooperativ, denn die Situation spitzt sich dadurch zu. Besser: Nutzen Sie notfalls Ihren Rest Selbstbeherrschung und beenden Sie das Gespräch ruhig und durchdacht. Sobald Sie ebenfalls aggressiv werden, haben Sie Ihrem Angreifer zu einem Sieg verholfen. Sie haben ihm die Gewissheit gegeben, dass er Sie aus der Fassung bringen kann.

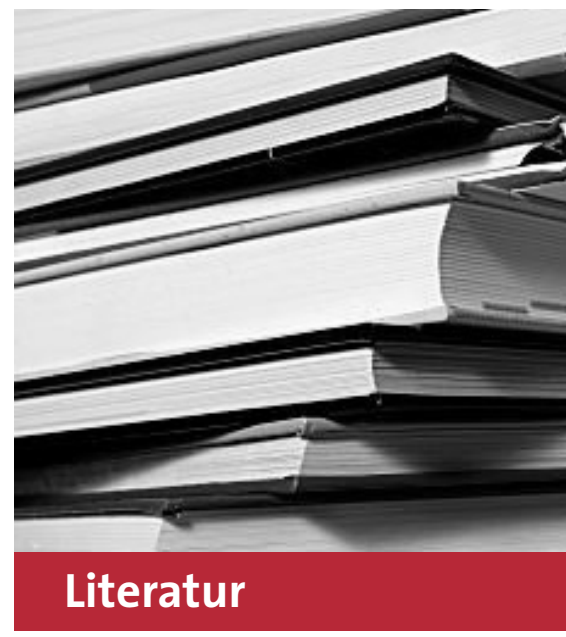

Schulz von Thun/Ruppel/Stratmann: Miteinander reden für Führungskräfte. 2000. S. 33-41. Internet http://www.friedenspaedagogik.de

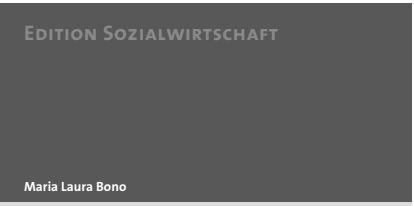

Performance Management in NPOs

Steuerung im Dienste sozialer Ziele

Domos

\section{Performance}

\section{Management in NPOs}

Steuerung im Dienste sozialer Ziele

Von Maria Laura Bono

2010, 221 S., brosch., 29,- $€$

ISBN 978-3-8329-5082-8

(Edition Sozialwirtschaft, Bd. 28)

Die Publikation setzt auf praxisnahe Ansätze, um in der sozialen Arbeit wirkungsorientiert zu steuern. Die Autorin stellt die Grundzüge des Performance Managements vor und analysiert die Bausteine eines Steuerungssystems. Ausgesuchte Fallbeispiele vertiefen zentrale Aspekte der NPOSteuerung und erleichtern die Entwicklung organisationsspezifischer Lösungen.

\section{Nomos}

